\title{
Conserved Densities for Linear Evolution Systems
}

\author{
L. Abellanas and A. Galindo \\ Department of Theoretical Physics, Universidad Complutense, Madrid-3, Spain
}

\begin{abstract}
A general discussion of the conservation laws for simple linear evolution systems is presented. The analysis is based upon an extension of the Gel'fand-Dikii symbolic algorithm to cover pseudo-differential operators. These techniques are applied to obtain all the conserved densities $\varrho[u]$ for the free Klein-Gordon and Dirac equations with nonzero mass.
\end{abstract}

\section{Introduction}

In previous papers [1-3] we have discussed the polynomial conserved densities for a single (linear or nonlinear) evolution equation. However, many other relevant equations in mathematical physics such as the Klein-Gordon equation, wave equation, and more generally, evolution systems like Dirac's were not covered by such analysis.

The aim of this paper is to present a full treatment of the conservation laws for simple (i.e. diagonalizable) linear evolution systems which greatly generalize older results [1]. This is accomplished by enlarging through Fourier techniques the Gel'fand-Dikii symbolic calculus [4] and allows us to use pseudo-differential operators. As an important byproduct, it is shown that all conserved densities for simple linear evolution systems are at most quadratic in the field variables barring the exceptional cases where one or more of the diagonalized evolution equations is of the form $v_{t}=(\mathbf{a} \cdot \mathbf{D}+b) v, \mathbf{a}, b$ constant. In particular, there it follows that for the free Klein-Gordon equation [5] and Dirac system with nonzero mass, any conserved density is quadratic in the fields.

In Sect. 2 we briefly expose the Fourier-Gel'fand calculus. Section 3 contains a detailed discussion of the conserved densities for simple linear evolution systems and Sect. 4 applies these results to linear evolution equations, to wave-like equations and to the Dirac system.

Appendix A contains two illustrative examples of some peculiar situations.

Finally, Appendix B establishes the relationship between linear evolution systems and individual partial differential equations (PDE's) for each field 
component. Such a result shows in particular that given a second order linear PDE, $u_{t t}=a(-i \mathbf{D}) u_{t}+b(-i \mathbf{D}) u$, an associated simple linear evolution system does generically exist which falls within the range of application of Sect. 3.

\section{A Fourier-Gel'fand Calculus for Pseudo-Differential Operators}

Let $\mathscr{U}$ stand for the set of $\mathbb{C}$-valued functions $u(\mathbf{x}, t), \mathbf{x} \in \mathbb{R}^{m}, t \in \mathbb{R}$, whose Fourier transform $\hat{u}(\mathbf{k}, t)$ is $C_{0}^{\infty}$ as a function of $\mathbf{k}$, and sufficiently smooth in $t$ (actually, $C^{\infty}$ in $t$ will serve our purposes). By $\mathscr{U}^{N}$ we shall denote the set of $N$-ples $\mathbf{u}=\left(u_{1}, \ldots, u_{N}\right)$, $u_{i} \in \mathscr{U}$.

Let $\mathscr{R} \equiv C^{\infty}\left(\mathbb{R}^{m}\right)$. Given two multi-indices $\alpha=\left(\alpha_{1}, \ldots, \alpha_{N}\right)=\alpha_{1} e_{1}+\ldots+\alpha_{N} e_{N}$, $\beta=\left(\beta_{1}, \ldots, \beta_{N}\right)=\beta_{1} e_{1}+\ldots+\beta_{N} e_{N}, \mathscr{R}_{\alpha, \beta}$ will stand for the set of $\mathbb{C}$-valued functions $r\left(k_{\alpha}^{\prime} ; k_{\beta}\right), C^{\infty}$ in the variables

$$
k_{\alpha}^{\prime} \equiv\left(\mathbf{k}_{11}^{\prime}, \ldots, \mathbf{k}_{1 \alpha_{1}}^{\prime}, \mathbf{k}_{21}^{\prime}, \ldots, \mathbf{k}_{2 \alpha_{2}}^{\prime}, \ldots, \mathbf{k}_{N 1}^{\prime}, \ldots, \mathbf{k}_{N \alpha_{N}}^{\prime}\right), k_{\beta} \equiv\left(\mathbf{k}_{11}, \ldots, \mathbf{k}_{N \beta_{N}}\right),
$$

and symmetric in the arguments $\left\{\mathbf{k}_{i 1}^{\prime}, \ldots, \mathbf{k}_{i \alpha_{i}}^{\prime}\right\}$, as well as in $\left\{\mathbf{k}_{j 1}, \ldots, \mathbf{k}_{j \beta_{j}}\right\}$, for all $i, j$.

The elements of $\mathscr{R}_{\alpha, \beta}$ will be called symbols.

Definition 1. Given $\mathbf{u} \in \mathscr{U}^{N}, r \in \mathscr{R}_{\alpha, \beta}$ we construct the following pseudodifferential map $\mathscr{U}^{N} \stackrel{r}{\longrightarrow} \mathscr{U}:$

$$
\begin{aligned}
\mathbf{u}(\mathbf{x}, t) \rightarrow(r[\mathbf{u}])(\mathbf{x}, t) \equiv & (2 \pi)^{-(|\alpha|+|\beta|) m / 2} \\
& \cdot \int r\left(k_{\alpha}^{\prime} ; k_{\beta}\right)\left(\Pi_{\alpha} \hat{u}^{*}\right)\left(k_{\alpha}^{\prime}, t\right)\left(\Pi_{\beta} \hat{u}\right)\left(k_{\beta}, t\right) \\
& \cdot\left[\exp i \mathbf{x} \cdot\left(\Sigma_{\beta} \mathbf{k}-\Sigma_{\alpha} \mathbf{k}^{\prime}\right)\right] d k_{\alpha}^{\prime} d k_{\beta},
\end{aligned}
$$

where

$$
\begin{gathered}
|\beta| \equiv \beta_{1}+\ldots+\beta_{N},\left(\Pi_{\beta} \hat{u}\right)\left(k_{\beta}, t\right) \equiv \prod_{i=1}^{N}\left[\prod_{j=1}^{\beta_{i}} \hat{u}_{i}\left(\mathbf{k}_{i j}, t\right)\right] \\
\Sigma_{\beta} \mathbf{k} \equiv \sum_{i=1}^{N}\left[\sum_{j=1}^{\beta_{2}} \mathbf{k}_{i j}\right], \quad d k_{\beta} \equiv \prod_{i=1}^{N}\left[\prod_{j=1}^{\beta_{i}} d^{m} k_{i j}\right]
\end{gathered}
$$

and similarly for the $\alpha$-terms.

Remarks. (i) The assumptions on $\mathscr{U}^{N}$ and $\mathscr{R}_{\alpha, \beta}$ guarantee that $r[\mathbf{u}] \in \mathscr{U}$. Therefore $r[\mathbf{u}]$ is $C^{\infty}$ and of fast decrease in $\mathbf{x}$, as well as smooth in $t$.

(ii) The above definition originates from the Gel'fand-Dikii symbolic calculus [4]. When $N=1$ and $r$ is a polynomial in $\mathscr{R}_{0, n}$, then $r[u]$ is just that homogeneous polynomial in $u$ and its spatial derivatives which has $(-i)^{n} r$ as its Gel'fand-Dikii symbol.

(iii) The term pseudo-differential operator has a precise meaning in the mathematical literature [5] on PDE's. The simplicity" of the differential systems envisaged in this paper has allowed us to relax some usual technical restrictions on the symbols $r$ as well as to extend the scope to nonlinear maps. 
Definition 2. Any finite linear combination

$$
\varrho[\mathbf{u}]=\sum_{i} r_{i}[\mathbf{u}], \quad r_{i} \in \mathscr{R}_{\alpha_{i}, \beta_{i}}
$$

will be called a density.

Since $r[\mathbf{u}]+r^{\prime}[\mathbf{u}]=\left(r+r^{\prime}\right)[\mathbf{u}]$ when $r, r^{\prime} \in \mathscr{R}_{\alpha, \beta}$, we will tacitly assume in the sequel that all $r_{i}$ entering (3) belong to different $\mathscr{R}_{\alpha_{l}, \beta_{i}}$.

Each density has an associated charge

$$
Q_{\varrho}(t)=\int(\varrho[\mathbf{u}])(\mathbf{x}, t) d^{m} x .
$$

Definition 3. Two densities $\varrho, \varrho^{\prime}$ are equivalent if their charges are equal:

$$
\varrho \sim \varrho^{\prime} \Leftrightarrow Q_{\varrho}=Q_{\varrho^{\prime}}, \quad \forall \mathbf{u}, t .
$$

If $\varrho \sim 0$, the density $\varrho$ will be called trivial.

Definition $3^{\prime}$. Two symbols $r, r^{\prime} \in \mathscr{R}_{\alpha, \beta}$ are equivalent $\left(r \sim r^{\prime}\right)$ if

$$
r\left(k_{\alpha}^{\prime} ; k_{\beta}\right)=r^{\prime}\left(k_{\alpha}^{\prime} ; k_{\beta}\right) \quad \text { on } \quad \Pi_{\alpha, \beta} \equiv\left\{k_{\alpha}^{\prime}, k_{\beta}: \Sigma_{\beta} \mathbf{k}=\Sigma_{\alpha} \mathbf{k}^{\prime}\right\} .
$$

If $r \sim 0$, the symbol $r$ will be called trivial.

Lemma 1. Given two symbols $r, r^{\prime} \in \mathscr{R}_{\alpha, \beta}$ and their associated densities $\varrho=r[\mathbf{u}]$, $\varrho^{\prime}=r^{\prime}[\mathbf{u}]$, the following assertions are equivalent:

(a) $r \sim r^{\prime}$

(b) $\varrho \sim \varrho^{\prime}$

(c) $\varrho-\varrho^{\prime} \in \operatorname{Ran} \mathbf{D}, \mathbf{D} \equiv\left(D_{x_{1}}, \ldots, D_{x_{m}}\right)$.

Proof. The equivalence a) $\Leftrightarrow$ b) is clear, since the exponential in (1) gives rise, upon integration in $\mathbf{x}$, to $\delta\left(\sum_{\beta} \mathbf{k}-\sum_{\alpha} \mathbf{k}^{\prime}\right)$.

Assertion (c) means that $\exists \mathbf{s}[\mathbf{u}], \mathbf{s}=\left(s_{1}, \ldots, s_{m}\right), s_{i} \in \mathscr{R}_{\alpha, \beta}$, such that $\varrho-\varrho^{\prime}$ $=\sum_{i} D_{x_{i}} s_{i}[\mathbf{u}]$, where $D_{x_{i}}$ denotes the total derivative with respect to $x_{i}$. Therefore, c) $\Rightarrow$ b) trivially. It will thus suffice to prove (a) $\Rightarrow\left(\right.$ c). But as $r, r^{\prime}$ are $C^{\infty}, r \sim r^{\prime}$ implies

$$
\left(r-r^{\prime}\right)\left(k_{\alpha}^{\prime} ; k_{\beta}\right)=\left(\Sigma_{\beta} \mathbf{k}-\Sigma_{\alpha} \mathbf{k}^{\prime}\right) \cdot \mathbf{s}^{i}\left(k_{\alpha}^{i} ; k_{\beta}\right)
$$

with $s_{j}^{\prime} \in \mathscr{R}_{\alpha, \beta}$. The choice $\mathbf{s}=-i \mathbf{s}^{\prime}$ completes the proof.

\section{Conserved Densities for Simple Linear Evolution Systems}

In this section we will apply the preceding formalism to obtain information on the conserved densities of some special evolution systems.

Let

$$
\mathbf{u}_{t}=A[\mathbf{u}], \quad \mathbf{u} \in \mathscr{U}^{N}
$$

denote a linear evolution system, where $A$ is a pseudo-differential matrix with entries $A_{i j} \in \mathscr{R}$. Since the entries of $\exp t A$ are also $C^{\infty}[6]$, the set $\mathscr{U}^{N}$ remains stable under the flow (7). 
Definition 4. The system (7) will be called simple if $\exists$ an inversible matrix $M$ which diagonalizes $A$, and such that the entries of both $M$ and $M^{-1}$ belong to $\mathscr{R}$ :

$$
M(\mathbf{k}) A(\mathbf{k}) M^{-1}(\mathbf{k}) \equiv L(\mathbf{k})=\operatorname{diag}\left\{l_{1}(\mathbf{k}), \ldots, l_{N}(\mathbf{k})\right\} .
$$

In terms of the new functions $\mathbf{v} \equiv M[\mathbf{u}],(7)$ can be reexpressed as a decoupled evolution system

$$
\mathbf{v}_{t}=L[\mathbf{v}] \text {, i.e. }\left(\hat{v}_{j}(\mathbf{k}, t)\right)_{t}=l_{j}(\mathbf{k}) \hat{v}_{j}(\mathbf{k}, t) .
$$

Definition 5. A density $\varrho[\mathbf{u}]$ is said to be conserved under (7) if

$$
\frac{d}{d t} Q_{\varrho}(t)=0, \quad \text { equivalently, } \quad D_{t} \varrho[\mathbf{u}] \sim 0
$$

for all $\mathbf{u} \in \mathscr{U}^{N}$ satisfying (7).

Any density $\varrho[\mathbf{u}]$ can be regarded as a density $\varrho[\mathbf{v}]$, and conversely. It is finally clear that $\varrho$ is conserved under (7) iff $\varrho$ is so under (9).

We shall denote by $C(A), C(L)$, respectively, the linear sets of conserved densities under (7), (9).

Lemma 2. A density $\varrho[\mathbf{v}]=\sum r_{i}[\mathbf{v}], r_{i} \in \mathscr{R}_{\alpha_{i}, \beta_{i}}$, is conserved under (9) iff each $r_{i}[\mathbf{v}]$ is conserved.

Proof. Due to the lack of coupling between different components $v_{j}$ in (9). if $\mathbf{v}(\mathbf{x}, t)$ is a solution of (9) the same is true for $\mathbf{v}_{\lambda}=\left(\lambda_{1} v_{1}, \ldots, \lambda_{N} v_{N}\right), \forall \lambda_{j} \in \mathbb{C}$. Let $\varrho_{\lambda}[\mathbf{v}] \equiv \varrho\left[\mathbf{v}_{\lambda}\right]$. Then

$$
Q_{\varrho_{\lambda}}(t)=\sum_{i} \lambda^{* \alpha_{i}} \lambda^{\beta_{i}} Q_{r_{i}}(t)
$$

with $\lambda^{\beta_{i}} \equiv \prod_{j=1}^{N} \lambda_{j}^{\beta_{i j}}$, etc. The constancy in $t$ of $Q_{\varrho_{\lambda}}(t)$ for all $\left(\lambda_{1}, \ldots, \lambda_{N}\right)$ requires $Q_{r_{i}}(t)$ to be constant.

The search of conserved densities for (9), and consequently for (7) is thus reduced to finding those symbols $r \in \mathscr{R}_{\alpha, \beta}$ such that $r[\mathbf{v}] \in C(L)$.

Proposition 1. Given $r \in \mathscr{R}_{\alpha, \beta}, r[\mathbf{v}] \in C(L)$ iff $r l_{\alpha, \beta} \sim 0$, where $l_{\alpha, \beta} \in \mathscr{R}_{\alpha, \beta}$ is

$$
l_{\alpha, \beta}\left(k_{\alpha}^{\prime} ; k_{\beta}\right) \equiv \sum_{i=1}^{N}\left[\sum_{j=1}^{\beta_{i}} l_{i}\left(\mathbf{k}_{i j}\right)+\sum_{j=1}^{\alpha_{i}} l_{i}^{*}\left(\mathbf{k}_{i j}^{\prime}\right)\right] \text {. }
$$

Proof. By definition, $r[\mathbf{v}]$ is conserved $\Leftrightarrow D_{t} r[\cdot] \sim 0$. Differentiating $r[\mathbf{v}]$ [as given in (1) after the substitution $u \rightsquigarrow v$ ] with respect to $t$ and using (9), one obtains

$$
D_{t} r[\cdot]=\left(r l_{\alpha, \beta}\right)[\cdot]
$$

which completes the proof.

The above proposition reduces the question of knowing which $r[\mathbf{v}], r \in \mathscr{R}_{\alpha, \beta}$, are conserved under (9) to finding those symbols $r$ such that $r l_{\alpha, \beta} \sim 0$. An exhaustive classification of possibilities is afforded by the following 
Proposition 2. Let the system (9) and $r \in \mathscr{R}_{\alpha, \beta}$ be given. Let $\mathscr{Z}\left(l_{\alpha, \beta}\right)$ denote the interior of the closed set of zeroes of $l_{\alpha, \beta}$ in $\Pi_{\alpha, \beta}$. Then:

(a) $\mathscr{Z}\left(l_{\alpha, \beta}\right)=\emptyset \Leftrightarrow \forall r[\mathbf{v}] \in C(L)$ are trivial.

(b) $\mathscr{Z}\left(l_{\alpha, \beta}\right)=\Pi_{\alpha, \beta} \Leftrightarrow \forall r[\mathbf{v}] \in C(L)$.

(c) $\emptyset \neq \mathscr{Z}\left(l_{\alpha, \beta}\right) \neq \Pi_{\alpha, \beta} \Leftrightarrow \exists$ nontrivial $r[\mathbf{v}] \in C(L)$, and $\exists r[\mathbf{v}] \notin C(L)$.

Proof. (a) $(\Rightarrow): \mathscr{Z}\left(l_{\alpha, \beta}\right)=\emptyset$ implies that $l_{\alpha, \beta} \neq 0$ on an open dense subset of $\Pi_{\alpha, \beta}$. Therefore, for $r[\mathbf{v}]$, to be conserved (i.e. $r l_{\alpha, \beta}=0$ on $\Pi_{\alpha, \beta}$, see Proposition 1) it is necessary that $r$ vanishes on such a dense subset. The continuity of $r$ makes $r \sim 0$.

$(\Leftarrow)$ : Suppose $\mathscr{Z}\left(l_{\alpha, \beta}\right) \neq \emptyset$. Then by choosing $r$ nontrivial, and with $\operatorname{suppr} \subset \mathscr{Z}\left(l_{\alpha, \beta}\right)$, we would get a nontrivial conserved density.

(b) $(\Rightarrow)$ : Trivial

$(\Leftarrow)$ : Trivial (take $r=1$ ).

(c) It follows from (a) and (b).

The possibility (b), i.e. $l_{\alpha, \beta} \sim 0$, is the most important, since it guarantees that the full set $\mathscr{R}_{\alpha, \beta}$ of symbols gives rise to conserved densities. We present next a systematic criterium to ascertain when $l_{\alpha, \beta} \sim 0$.

Criterium. The following statements hold:

(i) $l_{\alpha, 0} \sim 0, \alpha=e_{i} \Leftrightarrow l_{i}(\mathbf{0})=0$ $l_{0, \beta} \sim 0, \beta=e_{i} \Leftrightarrow l_{i}(\mathbf{0})=0$.

(ii) $l_{\alpha, 0} \sim 0, \alpha=e_{i}+e_{j} \Leftrightarrow l_{i}(\mathbf{k})+l_{j}(-\mathbf{k})=0, \forall \mathbf{k}$.

$l_{\alpha, \beta} \sim 0, \alpha=e_{i}, \beta=e_{j} \Leftrightarrow l_{i}^{*}(\mathbf{k})+l_{j}(\mathbf{k})=0, \forall \mathbf{k}$.

$l_{0, \beta} \sim 0, \beta=e_{i}+e_{j} \Leftrightarrow l_{i}(\mathbf{k})+l_{j}(-\mathbf{k})=0, \forall \mathbf{k}$.

(iii) $l_{\alpha, \beta} \sim 0,|\alpha|+|\beta| \geqq 3 \Leftrightarrow\left\{\begin{array}{l}l_{\alpha, \beta}(0 ; 0)=0 \\ l_{j}(\mathbf{k})-l_{j}(\mathbf{0})=-\left(l_{i}^{*}(\mathbf{k})-l_{i}^{*}(\mathbf{0})\right)=\lambda \cdot \mathbf{k}, \lambda \in \mathbb{C}^{m}, \forall \mathbf{k}, \forall i, j \\ \text { such that } \alpha_{i} \neq 0 \neq \beta_{j} .\end{array}\right.$

Proof. (i) and (ii) are self-evident. As to (iii), it suffices to prove the direct implication. Two essentially different cases can arise: $|\beta| \geqq 3$, or $|\beta| \geqq 2,|\alpha| \geqq 1$ (exchanging $\alpha$ with $\beta$ and $l_{i}$ with $l_{i}^{*}$ cover the remaining situations).

Let $|\beta| \geqq 3$. Then $l_{\alpha, \beta} \sim 0 \Rightarrow$

$$
\bar{l}_{h}\left(-\mathbf{k}_{1}-\mathbf{k}_{2}\right)+\bar{l}_{i}\left(\mathbf{k}_{1}\right)+\bar{l}_{j}\left(\mathbf{k}_{2}\right)=0, \quad \forall \mathbf{k}_{1}, \mathbf{k}_{2}
$$

whenever $\beta_{h}, \beta_{i}, \beta_{j} \neq 0$, and $\bar{f}(\mathbf{k}) \equiv f(\mathbf{k})-f(\mathbf{0})$. But (14) requires $\bar{l}_{h}=\bar{l}_{i}=\bar{l}_{j} \equiv \bar{l}, \bar{l}(\mathbf{k})$ $+\bar{l}(-\mathbf{k})=0$, so that (14) becomes

$$
\bar{l}\left(\mathbf{k}_{1}+\mathbf{k}_{2}\right)=\bar{l}\left(\mathbf{k}_{1}\right)+\bar{l}\left(\mathbf{k}_{2}\right)
$$

which expresses the additivity of $\bar{l}$. This property, together with the continuity of $\bar{l}$, forces $\bar{l}$ to be a linear function: $\bar{l}(\mathbf{k})=\boldsymbol{\lambda} \cdot \mathbf{k}$.

Now let $|\alpha| \geqq 1,|\beta| \geqq 2$. The assumption $l_{\alpha, \beta} \sim 0$ leads now to

$$
\bar{l}_{h}^{*}\left(\mathbf{k}_{1}+\mathbf{k}_{2}\right)+\bar{l}_{i}\left(\mathbf{k}_{1}\right)+\bar{l}_{j}\left(\mathbf{k}_{2}\right)=0, \forall \mathbf{k}_{1}, \mathbf{k}_{2}
$$

if $\alpha_{h}, \beta_{i}, \beta_{j} \neq 0$. Therefore $-\bar{l}_{h}^{*}=\bar{l}_{i}=\bar{l}_{j} \equiv \bar{l}$, with $\bar{l}$ fulfilling $(15)$, i.e. $\bar{l}(\mathbf{k})=\lambda \cdot \mathbf{k}$.

Since $|\alpha|+|\beta| \geqq 3$ ensures that one or several of the possibilities $|\alpha| \geqq 3,|\beta| \geqq 3,|\alpha|$ $\geqq 2$ and $|\beta| \geqq 1,|\beta| \geqq 2$ and $|\alpha| \geqq 1$, occurs, the proof is complete. 
The mixed possibility (c) in Proposition 2 is automatically discarded in the relevant case where all $l_{i}(\mathbf{k})$ are analytic functions of the real variable $k$. Consequently, we have:

Corollary 1. Assume $l_{i}$ analytic. Then according to whether $l_{\alpha, \beta}$ fulfills the preceding criterium or not, then either

or

(i) $\forall r[\mathbf{v}], r \in \mathscr{R}_{\alpha, \beta}$, belong to $C(L)$

(ii) $\forall r[\mathbf{v}] \in C(L), r \in \mathscr{R}_{\alpha, \beta}$, are trivial.

When the analyticity cannot be invoked, (c) is a real possibility (see Appendix A for a related example). However, it is rather remarkable that unless some $l_{i}$ are linear on certain open sets the system (9) admits nontrivial densities only when $|\alpha|$ $+|\beta| \leqq 2$. This is a mere consequence of the following lemma and Proposition 2.

Lemma 3. Let $f_{0}, f_{1}, \ldots, f_{n}, n \geqq 2$, be continuous functions of $\mathbf{k} \in \mathbb{R}^{m}$, satisfying

$$
f_{0}\left(\mathbf{k}_{1}+\ldots+\mathbf{k}_{n}\right)=f_{1}\left(\mathbf{k}_{1}\right)+\ldots+f_{n}\left(\mathbf{k}_{n}\right)
$$

for $\forall\left(\mathbf{k}_{1}, \ldots, \mathbf{k}_{n}\right) \in \Omega$ (nonvoid open subset of $\left.\left(\mathbb{R}^{m}\right)^{X n}\right)$. Then $f_{0}, f_{1}, \ldots, f_{n}$ are linear, with a common constant gradient, on some nonempty open subsets $\Omega_{0}$, $\Omega_{1}, \ldots, \Omega_{n} \subset \mathbb{R}^{m}$, respectively.

Proof. Take $\left(\overline{\mathbf{k}}_{1}, \ldots, \overline{\mathbf{k}}_{n}\right) \in \Omega$, and define $\tau_{i} \equiv \mathbf{k}_{i}-\overline{\mathbf{k}}_{i}, g_{i}\left(\tau_{i}\right) \equiv f_{i}\left(\mathbf{k}_{i}\right)-f_{i}\left(\overline{\mathbf{k}}_{i}\right), i=1, \ldots, n$, $g_{0}\left(\tau_{1}+\ldots+\tau_{n}\right) \equiv f_{0}\left(\mathbf{k}_{1}+\ldots+\mathbf{k}_{n}\right)-f_{0}\left(\overline{\mathbf{k}}_{1}+\ldots+\overline{\mathbf{k}}_{n}\right)$. Then $g_{0}(\mathbf{0})=g_{i}(\mathbf{0})=0$, and

$$
g_{0}\left(\tau_{1}+\ldots+\tau_{n}\right)=g_{1}\left(\tau_{1}\right)+\ldots+g_{n}\left(\tau_{n}\right)
$$

for $\forall \tau_{i}$ sufficiently small, say $\tau_{i} \in W_{2 \delta} \equiv\{\tau:|\tau|<2 \delta\}, \delta>0$. Therefore $g_{0}=g_{1}$ $=\ldots=g_{n} \equiv g$ on $W_{2 \delta}$, and the continuous function $g$ is additive on $W_{\delta}$. It is now simple to see that this additivity implies $g(a \mathbf{k})=a g(\mathbf{k})$ for all rational real numbers $a$ such that $\mathbf{k}, a \mathbf{k} \in W_{\delta}$. Continuity completes the proof.

Remark. If (17) holds for $\forall\left(\mathbf{k}_{1}, \ldots, \mathbf{k}_{n}\right)$, then one can take $\delta=\infty$ in the above proof. Therefore, Lemma 3 reproduces in particular the results of (iii) in the previous Criterium.

\section{Applications}

This section applies the results so far obtained to some interesting situations.

\subsection{Linear Evolution Equations}

Let the single linear equation

$$
u_{t}=l[u]
$$

with $l \in \mathscr{R}$. Proposition 2, Criterium, and Corollary 1 permit a full discussion of the conserved densities under (19). In particular, if $l$ is a polynomial $p$, we can immediately conclude the following result, which generalizes the final theorem of [1]: 
Proposition 3. Consider the equation $u_{t}=p[u], p$ a polynomial. Then:

(i) $p(0)=0 \Leftrightarrow \forall r[u], r \in \mathscr{R}_{0,1}, \mathscr{R}_{1,0}$ are conserved.

(ii) $p$ odd $\Leftrightarrow \forall r[u], r \in \mathscr{R}_{0,2}, \mathscr{R}_{2,0}$ are conserved.

(iii) $p$ pure imaginary $\Leftrightarrow \forall r[u], r \in \mathscr{R}_{1,1}$ are conserved.

(iv) $p(\mathbf{k})=\lambda \cdot \mathbf{k} \Leftrightarrow \forall r[u], r \in \mathscr{R}_{0, n}, \mathscr{R}_{n, 0}$ are conserved.

(v) $\left.\begin{array}{l}p(\mathbf{k})=a+i \mathbf{b} \cdot \mathbf{k}, \mathbf{b} \text { real } \\ n_{1} a^{*}+n_{2} a=0, n_{1}, n_{2} \neq 0\end{array}\right\} \Leftrightarrow \forall r[u], r \in \mathscr{R}_{n_{1}, n_{2}}$ are conserved.

Moreover, if $\exists$ a nontrivial conserved density $r[u], r \in \mathscr{R}_{n_{1}, n_{2}}$, then $s[u] \in C(p)$, for $\forall s \in \mathscr{R}_{n_{1}, n_{2}}$.

For instance, the free Schrödinger equation

$$
i u_{t}=-\Delta u, \quad \Delta \equiv \mathbf{D} \cdot \mathbf{D}
$$

has $p(\mathbf{k})=-i k^{2}$, which only satisfies the conditions (i), (iii). Therefore, the set $C(i \Delta)$ of conserved densities for the Schrödinger equation (20) is given (modulo trivial ones) by the linear span of $u, u^{*}$, and $u^{*} s[u], s \in \mathscr{R}$.

\subsection{Linear Wave Equations}

Consider the wave equation

$$
f_{t t}=q(-i \mathbf{D}) f_{t}+p(-i \mathbf{D}) f,
$$

where $f$ is a function of $\mathbf{x}, t$ and $p, q$ are polynomials in $\mathbf{D}$.

The substitution $f=u_{1}, f_{t}=u_{2}$ leads to the associated linear evolution system

$$
\mathbf{u}_{t} \equiv\left(\begin{array}{l}
u_{1} \\
u_{2}
\end{array}\right)_{t}=\left(\begin{array}{ll}
0 & 1 \\
p & q
\end{array}\right)\left(\begin{array}{l}
u_{1} \\
u_{2}
\end{array}\right) \equiv A[\mathbf{u}]
$$

When the discriminant $q^{2}(\mathbf{k})+4 p(\mathbf{k}) \neq 0, \forall \mathbf{k}$, that system is simple, and so the conserved densities for (21) [or equivalently (22)] can be readily obtained through Corollary 1 by inspecting the secular roots $l_{1}(\mathbf{k}), l_{2}(\mathbf{k}) \in \mathscr{R}$ of $A$. Suppose, for instance, the free massive Klein-Gordon equation

$$
f_{t t}=(\Delta-1) f \text {. }
$$

Now $l_{1}(\mathbf{k})=-l_{2}(\mathbf{k})=i\left[\left(1+k^{2}\right)^{1 / 2}\right]$. Let $\mathbf{v}$ be the field variables for the diagonalized system. Then Corollary 1 allows us to conclude that the set of conserved densities under (23) is, modulo trivial ones, linearly spanned by $r[\mathbf{v}], r \in \mathscr{R}_{\alpha, \beta}$, with $(\alpha, \beta)$ $=((0,0),(1,1)),((1,0),(1,0)),((0,1),(0,1)),((1,1),(0,0))$, once the variables $\mathbf{v}$ are reexpressed in terms of $\mathbf{u}$, i.e. $f, f_{t}$. A simple calculation shows that the set $C_{\mathrm{K} . G}$. of conserved densities for the free Klein-Gordon equation with non-zero mass is, modulo trivial densities, the linear span of the following quantities (whose origin from $\mathbf{v}$ is indicated):

$$
\begin{aligned}
& ((0,0),(1,1)) \rightarrow\left\{\begin{array}{l}
f_{t} s[f]-f_{s}\left[f_{t}\right] \\
f_{t} s\left[f_{t}\right]-f_{s}\left[f_{t t}\right],
\end{array}\right. \\
& \left.\begin{array}{l}
((1,0),(1,0)) \\
((0,1),(0,1))
\end{array}\right\} \rightarrow\left\{\begin{array}{l}
f_{t}^{*} s[f]-f^{*} s\left[f_{t}\right] \\
f_{t}^{*} s\left[f_{t}\right]-f^{*} s\left[f_{t t}\right],
\end{array}\right. \\
& ((1,1),(0,0)) \rightarrow\left\{\begin{array}{l}
f_{t}^{*} s\left[f^{*}\right]-f^{*} s\left[f_{t}^{*}\right] \\
f_{t}^{*} s\left[f_{t}^{*}\right]-f^{*} s\left[f_{t t}^{*}\right],
\end{array}\right.
\end{aligned}
$$


where $s \in \mathscr{R}$ is arbitrary. In the above expressions $f_{t t}$ should be replaced by the r.h.s. of (23).

Remark. Under the restriction $m \geqq 2$, it has been proved in [7] that (24) span $C_{\text {K.G. }}$. for the real case. The techniques used in [7] are radically different from ours, and their specificity to this example contrasts with the generality of the method presented here.

Should the mass be zero, i.e.

$$
f_{t t}=\Delta f
$$

the associated linear system is no longer simple if $m \geqq 2$, since the roots $l_{1}(\mathbf{k})=$ $-l_{2}(\mathbf{k})=i|\mathbf{k}|$ are not $C^{\infty}$ around $\mathbf{k}=0$. However, as $l_{i}(\mathbf{k})$ are linear nowhere, the remarks preceding Lemma 3 show that the set $C_{\text {K.G. }}(0)$ of conserved densities $r[\mathbf{u}]$ under the system (22) associated with (27) has not any nontrivial density with symbol $r \in \mathscr{R}_{\alpha, \beta},|\alpha|+|\beta| \geqq 3$. [Otherwise there would exist nontrivial densities of that type under the technical restriction that $\mathbf{u}$ vanish in some neighbourhood of $\mathbf{k}=0$, and for these, the lack of differentiability of $l_{i}(\mathbf{k})$ at the origin becomes irrelevant.] Regarding the case $|\alpha|+|\beta|=1$, it is straightforward to check that the only nontrivial $r[\mathbf{u}] \in C_{\text {K.G. }}(0), r \in \mathscr{R}_{\alpha, \beta}$, have $(\alpha, \beta)=((0,1),(0,0)),((0,0),(0,1))$. In summary, if $m \geqq 2, C_{\mathrm{K} . \mathrm{G} .} .(0)$ is linearly spanned, modulo trivial densities, by (24)(26) and $f_{t}, f_{t}^{*}$.

Let us finally consider (27), with $m=1$. Now it is possible to choose as secular roots $l_{1}(k)=-l_{2}(k)=i k$, which are analytic, odd and pure imaginary. However, once again the associated linear system is not simple. The application of the Criterium in Sect. 3 shows that $C(L)$ is spanned by $r[\mathbf{v}], r \in \mathscr{R}_{\alpha, \beta},(\alpha, \beta)=\left(\left(0, n_{1}\right)\right.$, $\left.\left(0, n_{2}\right)\right),\left(\left(n_{1}, 0\right),\left(n_{2}, 0\right)\right), \forall n_{1}, n_{2}$ with $n_{1}+n_{2} \geqq 1$. Since $v_{1}=f_{x}+f_{t}, v_{2}=f_{x}-f_{t}$, these densities will determine a subset of $C_{\text {K.G. }}(0)$. However, there may exist other conserved densities $\varrho \in C_{\mathrm{K} . G}(0)$ which cannot be obtained by this procedure, such as $\varrho=f^{*} f_{t}-f_{t}^{*} f$, unless the family of symbols is properly extended.

\subsection{Dirac System}

Let us finally consider the free Dirac equation in Hamiltonian form

$$
i \mathbf{u}_{t}=(-i \alpha \cdot \mathbf{D}+\mu \beta) \mathbf{u}, \quad \mu \neq 0,
$$

where $\alpha, \beta$ are the usual Dirac $4 \times 4$ matrices. Now

$$
A(\mathbf{k})=-i(\boldsymbol{\alpha} \cdot \mathbf{k}+\mu \beta) \equiv-i H_{0}(\mathbf{k})
$$

with $H_{0}$ the free Hamiltonian. It is well known that the Foldy-Wouthuysen transformation matrix (with $\gamma \equiv \beta \boldsymbol{\alpha}$ )

$$
M(\mathbf{k})=\cos \theta \cdot \mathbb{1}+\sin \theta \boldsymbol{\gamma} \cdot \mathbf{k}, \quad \theta \equiv \frac{1}{2} \tan ^{-1} \frac{|\mathbf{k}|}{\mu}
$$

diagonalizes $H_{0}(\mathbf{k})$, and $M(\mathbf{k}) A(\mathbf{k}) M^{-1}(\mathbf{k})=L(\mathbf{k})=\operatorname{diag}\{-i \omega(\mathbf{k}), \quad-i \omega(\mathbf{k}), i \omega(\mathbf{k})$, $i \omega(\mathbf{k})\}, \omega(\mathbf{k})=\left(k^{2}+\mu^{2}\right)^{1 / 2}$.

Since $\omega(\mathbf{0}) \neq 0$, and $\omega(\mathbf{k})$ is analytic but not linear anywhere, the Criterium shows that $r[\mathbf{v}] \in C(L), r \in \mathscr{R}_{\alpha, \beta}$, requires $|\alpha|+|\beta|=2$. More specifically, $C(L)$ is 
spanned, modulo trivial densities, by the following densities:

$$
\mathbf{v}^{*} \cdot\left(\begin{array}{ll}
a & 0 \\
0 & b
\end{array}\right)[\mathbf{v}], \quad \mathbf{v} \cdot\left(\begin{array}{ll}
0 & a \\
b & 0
\end{array}\right)[\mathbf{v}], \quad \mathbf{v}^{*} \cdot\left(\begin{array}{ll}
0 & a \\
b & 0
\end{array}\right)\left[\mathbf{v}^{*}\right]
$$

where $a, b$ are arbitrary $2 \times 2$ matrices with entries in $C^{\infty}$. A simple computation, using (30), brings the first term of (31) to the form

$$
\mathbf{u}^{*} \cdot\left[r_{1}+\mathbf{s}_{1} \cdot \mathbf{\Sigma}+r_{2} \beta+\mathbf{s}_{2} \cdot \beta \mathbf{\Sigma}, H_{0}\right]_{+}[\mathbf{u}],
$$

where $r_{i}, \mathbf{s}_{i}$ are $C^{\infty}$ and arbitrary. Similarly for the rest of (31), by merely replacing one of the u's in (32) by the charge conjugate spinor $\mathbf{u}^{c}$. This way we have obtained a linearly generating set for the set $C_{D}$ of conserved densities under (28), modulo trivial ones.

\section{Appendix A}

In this appendix we include a couple of examples which illustrate some special situations.

(i) Suppose the linear evolution equation $u_{t}=l[u]$. If $l(\mathbf{k})$ is not analytic, then the possibility (c) in Proposition 2 may arise. For instance, let

$$
u_{t}(x, t)=\sqrt{-i D} u(x, t),(x, t) \in \mathbb{R} \times \mathbb{R}
$$

which would appear in the process of diagonalization of $f_{t t}=-i f_{x}$. More concretely, $l(k)$ for (A.1) is

$$
l(k)= \begin{cases}|k|^{1 / 2}, & k \geqq 0 \\ i|k|^{1 / 2}, & k<0 .\end{cases}
$$

[Although $l \notin C^{\infty}$, we will relax here our technical assumptions for the sake of providing simple final expressions]. For $r[u]$ to belong to $C(l)$, with $r \in \mathscr{R}_{1,1}$, Proposition 1 requires

$$
\left.\begin{array}{rl}
|k|^{1 / 2} r(k ; k) & =0, \quad k \geqq 0 \\
\left(|k|^{1 / 2}-|k|^{1 / 2}\right) r(k ; k) & =0, \quad k<0
\end{array}\right\} .
$$

Thus any $r\left(k^{\prime} ; k\right)$ such that $r(k ; k)=0$ for $k \geqq 0$ will satisfy (A.3) and gives rise to a conserved density under (A.1). So, the choice $r\left(k^{\prime} ; k\right)=\left(k^{\prime}+k\right) \theta\left(-k^{\prime}-k\right)$ (again not sufficiently regular) leads to the following nontrivial conserved density for (A.1):

$$
(r[u])(x, t)=(2 \pi)^{-1} \int \frac{d y}{y+i 0}\left[u^{*}(x-y, t) u_{x}(x+y, t)-u_{x}^{*}(x-y, t) u(x+y, t)\right] .
$$

Moreover, the density $u^{*} u$ is not conserved. This shows that the cases (a) and (b) of Proposition 2 may not exhaust all possible situations.

(ii) The following example shows that the condition $l_{\alpha, \beta}(0 ; 0)=0$ may be determinant in some cases for the existence of conserved densities. Let

$$
\mathbf{u}_{t}(x, t)=\left(\begin{array}{cc}
9 D^{2}+D-6 & 9 D^{2}-9 D \\
-9 D^{2}-9 D & -9 D^{2}+D+3
\end{array}\right) \mathbf{u}(x, t) .
$$


The transformation

$$
\mathbf{v}=\left(\begin{array}{cc}
1+D & D \\
-D & 1-D
\end{array}\right) \mathbf{u}
$$

brings the simple system (A.5) to the diagonal form

$$
\mathbf{v}_{t}=\left(\begin{array}{cc}
D-6 & 0 \\
0 & D+3
\end{array}\right) \mathbf{v} \equiv L[\mathbf{v}] .
$$

Now $l_{1}(k)=i k-6, l_{2}(k)=i k+3$. From Corollary 1 and Proposition 2 it follows that $C(L)$, modulo trivial densities, is the linear span of $r[\mathbf{v}], r \in \mathscr{R}_{\alpha, \beta}, \alpha_{2}+\beta_{2}$

$=2\left(\alpha_{1}+\beta_{1}\right)$. This last restriction merely reflects the condition $l_{\alpha, \beta}(0 ; 0)=0$, since the remaining part of (iii) in the Criterium is automatically satisfied in this example. For instance $v_{1}^{n} v_{2}^{2 n} \in C(L), \forall n \geqq 1$. In terms of $\mathbf{u}, v_{1} v_{2}^{2}$ becomes the following conserved density under (A.5):

$\varrho=\left[\left(u_{1}+u_{2}\right)_{x}\right]^{3}+\left(u_{1}-2 u_{2}\right)\left[\left(u_{1}+u_{2}\right)_{x}\right]^{2}+\left(u_{2}^{2}-2 u_{1} u_{2}\right)\left(u_{1}+u_{2}\right)_{x}+u_{1} u_{2}^{2}$.

\section{Appendix B}

Let us consider the most general linear differential evolution system with $N=2$, $m=1$, namely

$$
\begin{aligned}
& f_{t}=\sum_{j} \alpha_{j} D^{j} f+\sum_{j} \beta_{j} D^{j} g \\
& g_{t}=\sum_{j} \gamma_{j} D^{j} f+\sum_{j} \delta_{j} D^{j} g
\end{aligned}
$$

with $\alpha_{j}, \beta_{j}, \gamma_{j}, \delta_{j} \in \mathbb{C}$. It is straightforward to prove, by differentiation, that both $f, g$ satisfy the same PDE:

$$
\begin{aligned}
\Omega(f) & =\Omega(g)=0 \\
\Omega & \equiv D_{t}^{2}-\sum_{j}\left(\alpha_{j}+\delta_{j}\right) D^{j} D_{t}+\sum_{j, k}\left(\beta_{j} \gamma_{k}-\alpha_{j} \delta_{k}\right) D^{j+k} .
\end{aligned}
$$

In the general case $\mathbf{u}_{t}=A[\mathbf{u}], \mathbf{u} \in \mathscr{U}^{N}, \mathbf{x} \in \mathbb{R}^{m}$, and $A$ with polynomial entries, it turns out again that each $u_{j}$ satisfies the same PDE:

$$
\begin{gathered}
\Omega_{A}\left(u_{j}\right)=0, \quad j=1, \ldots, N \\
\Omega_{A}=D_{t}^{N}-\sum_{j=0}^{N-1} a_{j}(-i \mathbf{D}) D_{t}^{j},
\end{gathered}
$$

where $a_{j}$ are polynomials. The reason for this remarkable property is the fact that the differential matrix $A$ annihilates its characteristic polynomial

$$
\operatorname{det}(\lambda \mathbb{1}-A) \equiv \lambda^{N}-\sum_{0}^{N-1} a_{j} \lambda^{j}
$$

whence, by applying the identity

$$
A^{N}-\sum_{0}^{N-1} a_{j} A^{j}=0
$$

to $\mathbf{u}$, (B.3) follows at once. 
Conversely, given a linear differential operator $\Omega\left(-\mathrm{iD}_{t},-\mathrm{i} \mathbf{D}\right)=D_{t}^{N}-\sum_{0}^{N-1}$ $\cdot a_{j}(-i \mathbf{D}) D_{t}^{j}$, it is always possible to find a linear differential evolution system $\mathbf{u}_{t}=A[\mathbf{u}]$ such that $\Omega=\Omega_{A}$. It suffices to take

$$
A=\left(\begin{array}{ccccc}
0 & 1 & 0 & \ldots & 0 \\
0 & 0 & 1 & \ldots & 0 \\
\vdots & \vdots & \vdots & & \vdots \\
a_{0} & a_{1} & a_{2} & \ldots & a_{N-1}
\end{array}\right) .
$$

The choice is of course not unique.

The case $N=2$ is the most important for the physical applications. In Sect. 4.2 we have seen that the associated linear system is generically simple, and therefore within the range of applicability of the general method exposed in Sect. 3.

Acknowledgements. The authors acknowledge some enlightening discussions with G. García Alcaine, F. Guil, and L. Martinez, as well as the financial support of the Instituto de Estudios Nucleares, J.E.N.

\section{References}

1. Abellanas, L., Galindo, A.: Lett. Math. Phys. 2, 399 (1978)

2. Abellanas, L., Galindo, A.: J. Math. Phys. (N.Y.) 20, 1239 (1979)

3. Abellanas, L., Galindo, A. : Submitted to the J. Math. Phys.

4. Gel'fand, I.M., Dikii, L.A.: Russian Math. Surv. 30: 5, 77 (1975)

5. Taylor, M.: Pseudodifferential operators. In: Lecture Notes in Mathematics, Vol. 416. Berlin, Heidelberg, New York: Springer 1974

6. Chevalley, C.: Theory of Lie groups. Princeton: Princeton University Press 1946

7. Tsujishita, T.: Lett. Math. Phys. 3, 445 (1979)

Communicated by H. Araki

Received November 7, 1979 
